\title{
Azacitidine for treatment of patients with myelodysplastic syndromes (MDS): practical recommendations of the German MDS Study Group
}

\author{
Katharina Götze • Uwe Platzbecker • Aristoteles Giagounidis • Detlef Haase • \\ Michael Lübbert • Carlo Aul • Arnold Ganser • Ulrich Germing • \\ Wolf-Karsten Hofmann
}

Received: 9 September 2009 / Accepted: 10 June 2010 /Published online: 22 June 2010

(C) Springer-Verlag 2010

\begin{abstract}
Myelodysplastic syndromes (MDS) are a group of common bone marrow disorders characterized by ineffective hematopoiesis, peripheral cytopenias, and a substantial risk of progression to acute myeloid leukemia (AML). For many years, the main treatment option for MDS was best supportive care which alleviates symptoms,
\end{abstract}

\author{
K. Götze $(\bowtie)$ \\ III. Medizinische Klinik, Technische Universität München, \\ Ismaningerstr. 15, \\ 81675 Munich, Germany \\ e-mail: k.goetze@lrz.tum.de \\ U. Platzbecker \\ Universitätsklinikum Carl Gustav Carus, \\ Dresden, Germany

\begin{abstract}
A. Giagounidis · C. Aul
St. Johannes Hospital,
\end{abstract} \\ Duisburg, Germany \\ D. Haase \\ Georg-August-Universität, \\ Göttingen, Germany \\ M. Lübbert \\ Albert-Ludwigs-Universität, \\ Freiburg, Germany \\ A. Ganser \\ Medizinische Hochschule, \\ Hannover, Germany \\ U. Germing \\ Heinrich-Heine-Universität, \\ Düsseldorf, Germany \\ W.-K. Hofmann \\ Universitätsmedizin Mannheim, \\ Mannheim, Germany
}

but has no effect on the natural course of the disease. Recently, demethylating agents have become available as a promising new treatment for patients with MDS. In two randomized clinical trials, the demethylating agent azacitidine has demonstrated a reduced risk of transformation to AML, improvement of peripheral blood values, an improved quality of life, and a definite survival advantage compared to conventional care regimens for patients with International Prognostic Scoring System score of intermediate-2 or high-risk MDS. This review aims to provide practical recommendations for the use of azacitidine and the management of its side effects in patients with MDS, assuring safe administration and best efficacy of treatment.

Keywords Myelodysplastic syndromes · MDS ·

Azacitidine $\cdot$ Epigenetic therapy $\cdot$ Demethylating agents

\section{Introduction}

Myelodysplastic syndromes (MDS) are clonal hematopoietic stem cell disorders characterized by dysplastic and inefficient hematopoiesis, peripheral cytopenias, and a substantial risk of progression into acute myeloid leukemia (AML). MDS belong to the more common bone marrow diseases, with an incidence in the general population of approximately five in every 100,000 individuals $[1,2]$. Moreover, the incidence of MDS increases sharply with age, reaching $20-50 / 100,000$ in the population aged $\geq 70$ years, and thus, making it the most frequently occurring hematological malignancy in this age group. With the exception of those patients who can successfully undergo allogeneic stem cell transplantation, MDS remains 
incurable. Thus, nearly all patients will eventually die from their disease, most often due to infectious complications or transformation to AML [3]. Improved treatment options for patients with MDS are therefore urgently needed.

The prognosis for individual MDS patients varies greatly and is dependent on several risk factors, including presence and type of chromosomal abnormalities, number of cytopenias, and percentage of bone marrow blasts. The current standard score for prognostication in MDS is the risk-based International Prognostic Scoring System (IPSS) [3]. Using this score, four distinctive risk groups can be identified, which differ in terms of overall survival and AML evolution (low, intermediate-1, intermediate-2, and high risk). MDS patients with a low or intermediate-1 IPSS score have a fairly favorable median survival of 5.7 and 3.5 years, respectively [3]. In contrast, patients with high-risk MDS (i.e., intermediate- 2 or high IPSS score) have a median survival of 1.2 or 0.4 years, respectively, and a high risk of rapid progression to AML [3]. Although the IPSS remains the most commonly employed score for assessment of prognosis in MDS, recently published studies have suggested that it might underestimate the prognostic importance of chromosomal abnormalities [4-6]. Moreover, the requirement for regular transfusions also represents an important negative prognostic factor not included in the IPSS [4].

Until recently, there has been an absence of satisfactory treatment options bridging the gap between best supportive care (BSC) on the one end and allogeneic stem cell transplantation (SCT) on the other end of the treatment spectrum. The only proven curative approach for MDS is allogeneic SCT [7-9], while other less intensive treatment strategies including non-intensive chemotherapy, immunomodulatory agents, or immunosuppressive therapy have not been able to change the natural course of the disease in high-risk MDS [10].

Demethylating agents such as azacitidine and 5-azadeoxycitidine (decitabine) have recently emerged as promising drugs for the treatment of MDS. The results of a Cancer and Leukemia Group B (CALGB) trial comparing azacitidine with BSC showed significantly improved remission rates with azacitidine compared to $\mathrm{BSC}$, as well as an improved progression-free survival and increased quality of life $[11,12]$. To specifically address the question of overall survival, a second large prospective randomized phase III trial comparing azacitidine to the three most commonly used treatments in higher-risk MDS was initiated, and has shown a definite survival advantage for patients treated with azacitidine [13].

The second available demethylating agent, decitabine, was also found to confer a benefit in terms of remission rates, as well as significantly prolonged progression-free survival in patients with high-risk MDS when compared to
BSC in a large prospective randomized clinical trial, although overall survival was not improved [14]. However, two other clinical studies with different decitabine treatment schedules and different patient risk cohorts have shown encouraging data that warrant further investigation of this drug in MDS $[15,16]$.

This review aims to provide practical recommendations for the use of azacitidine for the treatment of patients with MDS. The suggestions made here are based on evidence from recent randomized phase III trials, as well as the clinical experience of the authors in the practical use of this agent. They do not constitute formal guidelines but rather, represent consensus-based recommendations of the German MDS Study Group.

\section{Identification of patients for azacitidine treatment}

Azacitidine as initial therapy for high-risk MDS

In general, management of patients with MDS is dependent on IPSS score and transfusion requirement of the individual patient, as well as age and comorbidities. A frequent problem in daily clinical practice is missing data on bone marrow cytogenetics. In order to categorize an MDS patient by IPSS, a conventional cytogenetic profile, ideally with at least 20 evaluable metaphases, is required. If necessary, repeated bone marrow aspirates should be performed. Alternatively, fluorescence in situ hybridization analysis of bone marrow aspirates for the most common chromosomal abnormalities detected in MDS may be applied when conventional chromosome banding is not possible. Despite these efforts, some cases may remain where karyotype analysis cannot be obtained even after repeated bone marrow sampling. In these cases, patients with a bone marrow blast count of $11-20 \%$ may be considered to have high-risk MDS since they will have at least an Int-2 IPSS score, regardless of karyotype.

Age and comorbidities are especially relevant in the context of allogeneic stem cell transplantation. In particular, patients with high-risk MDS should be evaluated for allogeneic stem cell transplantation before treatment with azacitidine is considered. Those patients with high-risk MDS as defined by IPSS (intermediate- 2 or high), as well as patients with chronic myelomonocytic leukemia-II according to WHO, who are not eligible for allogeneic stem cell transplantation, generally, should be considered candidates for treatment with azacitidine. Patients fulfilling the criteria for refractory anemia with excess blasts in transformation (RAEB-T) according to the French-AmericanBritish (FAB) classification (20-29\% bone marrow blasts) may also be considered candidates for azacitidine treatment [17]. 
Azacitidine as treatment for low-risk MDS

Patients who remain IPSS Int-1 risk but display increasing transfusion requirement over time, as well as low-risk patients in whom other therapies (e.g., erythropoiesisstimulating agents, immunosuppressive therapy, immunmodulatory agents) have failed, should currently not be considered candidates for azacitidine treatment outside of a clinical trial. Although the efficacy and safety of azacitidine in lower-risk MDS has been established in a phase II open-label trial in the United States, a survival benefit has not been demonstrated for this subgroup of patients [18]. The authors feel that the potential benefit of azacitidine therapy in low-risk MDS may not outweigh the risk of harm through therapy-related adverse events such as neutropenia and thrombocytopenia. Therefore, a recommendation for the use of azacitidine in low-risk MDS outside of a controlled clinical trial cannot be made at this point.

\section{General considerations}

Clinical data have shown that response to azacitidine is independent of age [13]. More importantly, treatment of older patients $\geq 75$ years was not associated with increased toxicity [13]. Therefore, older age should not be an exclusion criterion for azacitidine therapy, and no upper age limit is defined. Comorbidities, which should be considered before initiation of therapy, include renal and hepatic impairment. Since azacitidine is primarily excreted by the kidneys, the risk of toxicity is expected to be greater in patients with impaired renal function, although no data from clinical studies are available on this. Likewise, the safety and pharmacokinetics of azacitidine have not been investigated in patients with evidence of hepatic impairment. It is therefore recommended that patients with renal or hepatic impairment be monitored closely. Patients with liver cirrhosis should not be treated with azacitidine. Azacitidine is contraindicated in patients with advanced malignant hepatic tumors. Azacitidine is potentially teratogenic. While this is not a concern for most elderly patients with MDS, women of childbearing potential should avoid becoming pregnant during therapy with azacitidine, and men should avoid fathering a child.

\section{Efficacy studies with azacitidine}

\section{CALGB 9221 trial}

The efficacy of azacitidine in the treatment of MDS has been evaluated in two phase II and two large randomized phase III clinical trials [11, 13, 19]. A total of 191 MDS patients were enrolled into the first phase III CALGB 9221 trial [11]. Patients were randomized to receive $\mathrm{BSC}$ or azacitidine by subcutaneous injection at a dose of $75 \mathrm{mg} / \mathrm{m}^{2}$ on seven consecutive days every 28 days. All subtypes of MDS could be enrolled. However, patients with MDS subtypes refractory anemia/RA with ringed sideroblasts were included only if they had severe thrombocytopenia, or neutropenia, or transfusion dependent anemia. After a minimum of 4 months of supportive care, patients whose disease progressed were permitted to cross over to azacitidine treatment. A total of 99 patients were primarily treated with azacitidine, and an additional 49 patients $(55 \%$ of patients in the observation arm) crossed over to azacitidine after 4 months. Approximately half of the total patient population had high-risk MDS as determined by IPSS criteria. The primary endpoint of the study was overall response rate.

According to the International Working Group (IWG) criteria for assessing responses in MDS [20,21], the overall response rate in the azacitidine arm was 47\% [19]. Responses to azacitidine were observed in all FAB subtypes. The majority of patients showed hematologic improvement, while $11 \%$ achieved either a complete remission (CR) or partial remission (PR). There were no complete or partial remissions in the observation arm, but $17 \%$ of patients also showed hematologic improvement (HI) with BSC. Patients treated with azacitidine showed a significant delay in progression to AML compared to patients in the BSC arm (21 vs. 13 months, $p=0.007)$. Improved survival by azacitidine treatment could not be established due to the crossover design and cohort size. However, an absolute difference in survival of 6 months in favor of azacitidine (20 months vs. 14 months for BSC) was documented, suggesting a possible survival benefit.

Among the 99 patients randomized to azacitidine, $66 \%$ were transfusion dependent at baseline. Of these, $45 \%$ had an elimination of all transfusions, while another $9 \%$ had a reduction in transfusions by $50 \%$ [19]. In addition, lineage responses for platelets and white blood cells (WBC) occurred in $47 \%$ and $40 \%$, respectively, among the patients treated with azacitidine. The median duration of transfusion independence was 9 months.

Further studies conducted separately on the CALGB 9221 patient population documented an improved quality of life for those MDS patients treated with azacitidine [12]. This was evidenced by the reduction of transfusion requirements, decreased rate of infections and hospitalizations, as well as improvement of fatigue, dyspnea, and physical functioning in patients receiving azacitidine.

\section{AZA-001 trial}

To definitely determine whether treatment with azacitidine conferred a survival benefit to MDS patients, a second large 
randomized phase III trial, with survival as primary endpoint, was initiated. The AZA-001 trial included 358 patients with high-risk MDS (Int-2 or high-risk according to IPSS) [13]. Patients were randomized to receive either azacitidine (179 patients) at a dose of $75 \mathrm{mg} / \mathrm{m}^{2}$ for 7 days, or one of three conventional care regimens (CCR). The choice of CCR included BSC (105 patients), low-dose cytarabine (49 patients), or intensive "AML-like" induction chemotherapy ( 25 patients) as selected by investigators before randomization. This trial showed a remission rate $(\mathrm{CR}+\mathrm{PR})$ of $29 \%$ for azacitidine compared to $12 \%$ for CCR $(p<0.0001)$ and a hematological improvement rate of $49 \%$ for azacitidine compared to $29 \%$ for CCR $(p<0.0001)$. Transfusion independence was achieved in $45 \%$ of patients receiving azacitidine. More importantly, a significantly prolonged median overall survival for the azacitidine group compared to the CCR group was demonstrated (24.5 vs. 15.0 months, respectively, $p=0.0001$ ). In addition, time to AML progression as a secondary study endpoint was significantly increased in the azacitidine arm (17.8 vs. 11.5 months, $p<0.0001)$. The absolute survival benefit for patients treated with azacitidine compared to CCR was 9.4 months. The percentage of patients alive at 2 years was twofold higher in the azacitidine arm as compared to the CCR arm (azacitidine, 51\%; CCR, 26\%; $p<0.0001$ ). Data on patients with sAML according to WHO criteria (FAB RAEB-T) included in the AZA-001 have recently been analyzed, and found to also confer a survival benefit to this subgroup of patients [17].

\section{Safety and tolerability of azacitidine}

The most common adverse event occurring during treatment with azacitidine is myelosuppression. Since cytopenias are inherent to MDS, it is difficult to assess to which extent myelosuppression is therapy-related or part of the natural course of the disease. However, low baseline blood cell counts further decreased in patients receiving treatment with azacitidine. Accordingly, the most common reasons for treatment discontinuation, dose reduction or interruption were leukocytopenia, neutropenia, and thrombocytopenia. In the CALGB 9221 trial, grade 3-4 leukocytopenia occurred in $59 \%$, granulocytopenia in $81 \%$, and thrombocytopenia in $70 \%$ of patients receiving azacitidine [11]. Infection thought to be related to treatment was reported in $20 \%$ of patients. The highest rate of adverse events was reported during the first two cycles of therapy and decreased in subsequent cycles. The proportion of patients with adverse events did not increase with patient age. There was one treatmentrelated death $(<1 \%)$ in the CALGB 9221 trial, and four treatment-related deaths $(2 \%)$ in the AZA-001 trial. In the AZA-001 trial, rates of grade 3-4 toxicity were $91 \%$ for neutropenia, $85 \%$ for thrombocytopenia, and 57\% for anemia [13]. Five percent of patients receiving azacitidine discontinued treatment in the AZA-001 trial before study completion due to hematological adverse events.

Nonhematological adverse events were most commonly either gastrointestinal or were associated with subcutaneous administration. Common gastrointestinal side effects include nausea, vomiting, diarrhea, constipation, and anorexia. Other reported adverse events were arthralgia, cough, headache, weakness, dizziness, and insomnia. Liver function abnormalities occurred in patients with intercurrent illnesses, and more severe abnormalities were noted in patients with liver cirrhosis [22].

\section{Predictive factors for treatment with azacitidine}

\section{Chromosomal abnormalities}

Recurring cytogenetic abnormalities associated with MDS can be divided into good, intermediate, and poor risk categories [3, 6]. Patients with good risk karyotypes (normal karyotype, isolated deletions of chromosome 20 (del(20q)), chromosome $5(\operatorname{del}(5 q))$, or loss of the Y chromosome as sole abnormality) have a more favorable prognosis. Those high-risk MDS patients who display a normal karyotype may occasionally benefit from intensive "AML-like" chemotherapy and achieve long-term remissions [23, 24]. Conversely, MDS patients with poor risk cytogenetics (complex aberrant karyotypes and/or alterations involving chromosome 7) have a very unfavorable prognosis $[6,25,26]$. These patients generally do not respond to conventional cytotoxic agents, making them poor candidates for intensive chemotherapy [27].

Demethylating agents have been shown to be beneficial in MDS with adverse cytogenetics [28-31]. A high rate of remission in MDS patients with chromosome 7 abnormalities treated with azacitidine was first observed in two smaller uncontrolled studies $[30,32]$. In the randomized AZA-001 trial, treatment with azacitidine was shown to be effective in all cytogenetic subgroups of MDS. In particular, a high response rate was observed in the group of patients with chromosome 7 abnormalities [33,34]. Survival in this subgroup was significantly prolonged in patients receiving azacitidine compared to CCR (13.1 months vs. 4.6 months, $p=0.002)$, both in the group of patients with $-7 / \operatorname{del}(7 q)$ alone as well as the group with $-7 / \operatorname{del}(7 q)$ as part of a complex aberrant karyotype [34]. Furthermore, in a large retrospective analysis of the outcome of MDS patients with chromosome 5 or 7 abnormalities, treatment with azacitidine or decitabine was superior to conventional chemotherapy in terms of survival [31]. Thus, azacitidine has a high efficacy in this subgroup of MDS patients with a very poor prognosis. 
MDS patients with intermediate or good prognostic karyotypes in the AZA-001 trial also had an increased survival with therapy with azacitidine as compared to CCR. The median overall survival for the azacitidine arm was not reached in the good risk cytogenetic subgroup, and was 26.3 months in the intermediate risk cytogenetic subgroup compared to 17 months for both cytogenetic groups in the CCR arm ( $p=0.04$ and 0.02 , respectively)[13].

Other factors

Response to azacitidine was not influenced by age, sex, MDS subtype by FAB or WHO classification, LDH serum levels, or percentage of bone marrow blasts $[13,19]$.

\section{Practical recommendations regarding treatment with azacitidine}

The standard dosing regimen for azacitidine employed in the CALGB 9221, as well as the AZA-001 trial is $75 \mathrm{mg} / \mathrm{m}^{2}$ for seven consecutive days repeated every 28 days (AZA-7). Patients should be premedicated to prevent nausea and vomiting. Azacitidine is administered subcutaneously. The starting dose of $75 \mathrm{mg} / \mathrm{m}^{2}$ is recommended regardless of baseline blood values. Since the 7-day regimen is inconvenient in the outpatient setting, alternative schedules, either skipping weekends (AZA-5-2) or administering azacitidine for only 5 days (AZA-5) have been proposed. Recently published data from an open-label randomized phase II trial have demonstrated comparable efficacy of these two schedules with the established 7-day schedule [18], although overall survival was not a study endpoint, and the original 7-day regimen was not included in this study. However, the majority of patients $(63 \%)$ included in this trial had low-risk MDS. The use of the AZA-5 schedule results in a 30\% dose reduction compared to the AZA-7 or AZA-5-2 schedule. Accordingly, toxicity in terms of grade 3-4 hematologic adverse events was lower using the AZA-5 than the AZA-52 regimen. It is conceivable that a decreased dose may also translate into reduced efficacy in high-risk MDS patients. Therefore, the use of a dose-reduced AZA schedule (if not necessitated by hematological toxicity) cannot be recommended in the high-risk setting.

\section{Time to response}

Due to its proposed mode of action of promoting demethylation in newly synthesized DNA, several rounds of DNA replication are necessary for azacitidine to elicit a full effect [35-37]. Thus, clinical responses do not occur immediately. In the CALGB 9221 trial, the median number of cycles from the first treatment with azacitidine to response was three cycles (range, one to 17 cycles) [19]. Although $75 \%$ of responding patients achieved a response by cycle four, one-fourth of responding patients showed an effect as late as cycle 17 . The majority of responding patients $(90 \%)$ achieved a response by the sixth cycle. Similarly, in the AZA-001 trial, best response was achieved after a median of four cycles, with an $87 \%$ probability of patients achieving a response by cycle six. On the basis of these results, it becomes evident that terminating therapy, if no response is seen after a few cycles, would be premature and would prevent many patients from benefiting from azacitidine treatment. We recommend treating MDS patients with azacitidine for a minimum of six cycles before evaluating response, unless overt progression to AML or unacceptable toxicity occurs. This seems to be the most practical approach although it will undoubtedly lead to some late responders being missed. In daily clinical practice, the decision whether or not to continue azacitidine treatment in the absence of response after six cycles is difficult and must be made on an individual basis, which includes consideration of the patient's preference.

\section{Optimum duration of therapy}

Another question at issue is the optimal duration of azacitidine therapy in patients showing any response. In the CALGB 9221 trial, the range of cycles was $1-17$, but a median cycle number was not stated [11]. Best response lasted for a median of five cycles after achievement of first response [19]. In the AZA-001 trial, cycle range was 1-39 with a median of nine cycles [13]. Continuation of azacitidine treatment led to a higher IWG response category in $48 \%$ of responding patients, and these patients received a median of eight additional azacitidine courses [38]. The median duration of hematological response (CR, PR, or any hematologic improvement) in the AZA-001 trial was 13.6 months. In a small retrospective study, the outcome of patients with high-risk MDS, treated with a maximum number of six azacitidine courses, was analyzed [39]. Patients were treated for four courses, with two additional courses administered as consolidation if a response was seen after four cycles. Although patients clearly benefited even from this limited number of treatment courses, remission rates were lower, and median survival was inferior to the AZA-001 trial where treatment was continued until progression.

The optimal duration of therapy has not been clearly defined and should be a focus of further studies. In the absence of unacceptable toxicity, the authors recommend continuation of azacitidine treatment in all patients achieving $\mathrm{CR}, \mathrm{PR}$, or HI until progression. Since a direct comparison 
between patients achieving stable disease with azacitidine vs. conventional care in the AZA-001 study does not exist due to low patient numbers in this category, the authors feel there is insufficient data to make a definite recommendation in this case. The decision to continue therapy in patients with stable disease should be at the treating physician's discretion and take into account whether or not the patient is motivated to continue therapy. It is unclear whether treatment with azacitidine beyond progression may still be able to slow down progression kinetics. Although there may be a lack of treatment options in these cases, the authors strongly suggest that azacitidine should be discontinued when clear evidence of progression has been established. There are insufficient data to draw definite conclusions on re-challenging patients with azacitidine at relapse, although anecdotal reports suggest this may be feasible. It has been reported that patients who have become refractory to azacitidine may benefit from a switch to decitabine [40].

Table 1 summarizes recommendations for duration of therapy.

\section{Response assessment}

Most patients responding to azacitidine achieve improvement of their baseline blood values and about half of them become transfusion independent. This form of response is easily determined by observing blood counts over time. Peripheral blood counts often worsen initially, especially during the first two cycles of therapy, before they improve in responding patients. This is one of the reasons for deferring response assessment until cycle six. To accurately assess PR or CR, as well as cytogenetic remissions, bone marrow evaluation is necessary. If response to azacitidine is evident by improving blood values and decreased transfusion requirement, additional bone marrow evaluation may be considered optional in patients with normal karyotype MDS. However, in the case of stable blood values, bone marrow evaluation provides important additional information, i.e., on the reduction of blast counts. In patients with abnormal cytogenetics, assessment of response by bone marrow aspirate is recommended to enable determination of cytogenetic remission. Moreover, patients should not be categorized as non-responders on the basis of peripheral blood values alone. Bone marrow evaluation may reveal a response in terms of a decrease in blasts even if peripheral blood counts remain unchanged. Improved quality of life, as reported by patients, may also be a useful parameter in determining benefit from azacitidine therapy, especially if only disease stabilization is observed.

\section{Management of side effects}

In general, side effects tend to occur more often during the first two cycles of azacitidine therapy, and then taper off with the use of appropriate concomitant medications [22]. However, adverse events may occur at any time and patients should be closely monitored on an outpatient basis even when they are between cycles. Adverse events should be actively managed, particularly during the first two cycles to allow continuation of treatment with azacitidine and thus, achievement of maximum therapeutic effect. Table 2 summarizes adverse events and their recommended management.

\section{Cytopenias}

Monitoring of complete blood counts is necessary throughout treatment. Since virtually all MDS patients have cytopenia in at least one hematopoietic lineage, assessing the myelosuppressive effect of azacitidine remains difficult. Dose reduction is required most often for severe neutropenia or thrombocytopenia, but discontinuation of therapy due to hematological toxicity is almost never necessary. In the AZA-001 trial, only $14 \%$ of patients required dose adjustments for adverse hematologic events and $71 \%$ of these were manageable with a single dose reduction. More often, transient worsening of cytopenia can be managed by delaying the next treatment cycle, or by concurrent transfusion of red blood cells or platelets. Importantly, if the grade

Table 1 Recommendations for duration of azacitidine treatment

\begin{tabular}{lc}
\hline Initial treatment & Recommendations \\
Patients who achieve CR, PR, or $\mathrm{HI}^{\mathrm{a}}$ & $\begin{array}{c}\text { Continue treatment with azacitidine for at least } 6 \text { cycles before evaluation of response, } \\
\text { unless unacceptable toxicity or progression to overt leukemia occurs } \\
\text { If CR, PR, or HI was achieved after } 6 \text { cycles, continue treatment with azacitidine to maximize } \\
\text { benefit for as long as patient has documented response, and is willing to continue treatment } \\
\text { Patients who achieve disease stabilization }{ }^{\text {a }}\end{array}$ \\
$\begin{array}{c}\text { If SD is achieved after } 6 \text { cycles, decision to continue treatment with azacitidine must be made } \\
\text { on individual basis, as there are insufficient data to make a definite recommendation }\end{array}$ \\
\hline
\end{tabular}

$C R$ complete remission, $P R$ partial remission, $H I$ hematologic improvement, $S D$ stable disease

${ }^{a}$ Modified International Working Group (IWG) 2000 criteria for myelodysplastic syndromes [21] 
Table 2 Common side effects of azacitidine and recommendations for management

\begin{tabular}{|c|c|c|c|}
\hline Adverse event & Monitoring & Prophylaxis & Therapy \\
\hline Hematologic & $\mathrm{CBC}$ at regular intervals & $\begin{array}{l}\text { Consider G-CSF if expected } \\
\text { neutropenia exceeds } 10 \text { days }\end{array}$ & $\begin{array}{l}\text { Delay next cycle until recovery of CBC } \\
\text { Reduce dose in next cycle if blood values do } \\
\text { not recover within } 2 \text { weeks of designated } \\
\text { day } 1 \text { of next cycle } \\
\text { Transfusion of RBC and platelets as required }\end{array}$ \\
\hline Infection & $\begin{array}{l}\text { Regular clinical examination } \\
\text { Educate patient to seek medical } \\
\text { care promptly if temp }>38.5^{\circ} \mathrm{C} \\
\text { occurs }\end{array}$ & $\begin{array}{l}\text { Consider G-CSF in following cycles } \\
\text { Consider antibiotics (e.g., quinolones) }\end{array}$ & $\begin{array}{l}\text { Antibiotics following guidelines } \\
\text { for neutropenic fever }\end{array}$ \\
\hline Nausea and emesis & & $\begin{array}{l}\text { Premedicate with antiemetics } \\
\text { (metoclopramide, alizapride } \\
\text { or 5-HT3 antagonist) }\end{array}$ & $\begin{array}{l}\text { Escalate antiemetic regimen } \\
\text { (5-HT3 antagonist, dexamethasone) }\end{array}$ \\
\hline Diarrhea & & & $\begin{array}{l}\text { I.V. fluids } \\
\text { Loperamide }\end{array}$ \\
\hline Constipation & & $\begin{array}{l}\text { Consider laxatives when using high } \\
\text { dose 5-HT3 antagonists }\end{array}$ & Laxatives, stool softener \\
\hline \multirow[t]{2}{*}{$\begin{array}{l}\text { Injection site } \\
\text { reaction }\end{array}$} & Clinical examination & Correct injection technique & $\begin{array}{l}\text { Symptomatic (evening primrose oil, } \\
\text { cooling compresses, soothing lotion) }\end{array}$ \\
\hline & & Rotation of injection sites & Topical steroids \\
\hline
\end{tabular}

$C B C$ complete blood count, $R B C$ red blood cell, $G-C S F$ granulocyte-colony stimulating factor

of cytopenia increases in one hematopoietic cell line but improves in another, the next azacitidine cycle should not be delayed and no dose adjustment should be made. In the AZA-001 trial, the temporary increase in cytopenias did not translate into an increased rate of infection or bleeding, and did not adversely affect response or survival [13].

Use of antibiotics and growth factors in febrile neutropenia and infection

Although anemia and thrombocytopenia can be expected to improve over the course of azacitidine therapy in responding patients, correction of existing neutropenia is less common [13]. The risk of serious infection is increased in patients with neutropenia, and infection is a common cause of death in MDS. Infectious complications during treatment with azacitidine occur most commonly in neutropenic patients and are potentially dangerous, though fatal outcomes have rarely been reported. Patients should receive clear instructions to seek medical attention promptly if febrile neutropenia occurs. Management of febrile neutropenia in patients treated with azacitidine is no different than with other cytotoxic drugs and should follow established guidelines, including prompt initiation of empirical antibiotics.

Prophylactic antibiotics such as quinolones are not always effective in preventing infection in patients with MDS and are therefore not used routinely. However, in patients experiencing febrile neutropenia during azacitidine treatment, prophylactic use of antibiotics in subsequent treatment cycles is appropriate. For patients with persistent neutropenia despite improvement in platelet counts or hemoglobin levels, concurrent administration of granulocyte colony-stimulating factor (G-CSF) may be considered. However, a positive effect of G-CSF in this setting has not been established.

\section{Gastrointestinal adverse effects}

Gastrointestinal side effects are common but can generally be managed easily with appropriate medication. Premedication for prevention of nausea and vomiting is necessary. For the most part, administration of metoclopramide or a 5HT3 antagonist prior to azacitidine is sufficient. However, in some cases, nausea may be more severe and additional antiemetic medication may be required. Corticosteroids such as dexamethasone are effective, but should be used with caution as they may increase existing immunosuppression. Diarrhea or constipation may occur and constipation is more frequent with the use of 5-HT3 antagonists. Generally, diarrhea may be controlled with antidiarrheal agents such as loperamide hydrochloride, while constipation may be managed with stool softeners and laxatives.

Injection site reactions

Injection site reactions in the form of localized erythema or bruising occur in almost all patients with subcutaneous application of azacitidine. Generally, these reactions are transient and do not require specific therapy. Local skin reactions can be minimized by using a fresh needle for each 
application and not purging the air from the needle before injection. In addition, local application of evening primrose oil (Oenothera biennis) directly after injection has recently been shown to alleviate inflammatory skin reactions [41]. Injection sites should be rotated, and new injections should never be given into areas where the site is tender, bruised, red, or hard. In some cases, pruritus, inflammation, or induration of the injection site may occur requiring more attention. Application of a cool compress, local application of steroids, or a soothing lotion (e.g., chamomile) may provide symptomatic relief. Occasionally, severe skin reactions may necessitate switching to intravenous administration.

\section{Future directions}

\section{Azacitidine at relapse after allogeneic SCT}

Patients with high-risk MDS or AML relapsing after allogeneic SCT have limited therapeutic options. As a novel strategy to maximize the graft-versus-leukemia effect, several investigators have administered azacitidine for relapse after allogeneic SCT. Successful treatment with azacitidine followed by donor lymphocyte infusion (DLI) was recently reported for a patient with leukemic relapse of MDS after allogeneic SCT, resulting in complete remission [42]. Furthermore, encouraging results using this strategy have been shown in a large pilot study of lower dose azacitidine in combination with DLI for elderly patients with relapsed AML or CMML after allogeneic SCT, paving the way for a prospective clinical trial [43]. Similarly, in a small uncontrolled study, administration of low dose azacitidine as a single agent at relapse after allogeneic SCT resulted in a high response rate in patients with AML [44]. Azacitidine has also been used successfully within a clinical trial as pre-emptive treatment to prevent full-blown relapse in patients with AML and minimal residual disease after allogeneic SCT [45].

\section{Combination therapies}

Ongoing and future studies will evaluate the potential of combining azacitidine with other novel agents to further improve outcome in patients with MDS. One attractive approach is to combine azacitidine with a histone deacetylase (HDAC) inhibitor to maximize epigenetic modulation of DNA. In addition to valproate and all-trans retinoic acid (ATRA), both HDAC inhibitors, which have been available for many years, newer and more potent HDAC inhibitors have recently been developed and are entering clinical trials [46-49]. In high-risk MDS patients, concomitant therapy with azacitidine and the immunomodulatory agent lenalidomide has been shown to be feasible without increased toxicity in a phase I trial [50]. In low-risk MDS, azacitidine, in combination with erythropoiesis-stimulating agents, is also being assessed. These innovative clinical trials will hopefully lead the way for new and effective treatment options for MDS patients in the future.

Acknowledgements This work was supported by the German Kompetenznetz "Akute and chronische Leukämien".

Disclosure of potential conflicts of interest All authors report having served on an advisory board for Celgene within the past 24 months, as well as having received honoraria for lectures from Celgene in the past.

\section{References}

1. Germing U, Aul C, Niemeyer CM et al (2008) Epidemiology, classification and prognosis of adults and children with myelodysplastic syndromes. Ann Hematol 87:691-699

2. Rollison DE, Howlader N, Smith MT et al (2008) Epidemiology of myelodysplastic syndromes and chronic myeloproliferative disorders in the United States, 2001-2004, using data from the NAACCR and SEER programs. Blood 112:45-52

3. Greenberg P, Cox C, LeBeau MM et al (1997) International scoring system for evaluating prognosis in myelodysplastic syndromes. Blood 89:2079-2088

4. Malcovati L, Porta MGD, Pascutto C et al (2005) Prognostic factors and life expectancy in myelodysplastic syndromes classified according to WHO criteria: a basis for clinical decision making. J Clin Oncol 23:7594-7603

5. Park MJ, Kim HJ, Kim SH et al. (2008) Is International Prognostic Scoring System (IPSS) still standard in predicting prognosis in patients with myelodysplastic syndrome?: External validation of the WHO Classification-Based Prognostic Scoring System (WPSS) and comparison to IPSS. Eur J Haematol.

6. Haase D, Germing U, Schanz J et al (2007) New insights into the prognostic impact of the karyotype in MDS and correlation with subtypes: evidence from a core dataset of 2124 patients. Blood 110:4385-4395

7. de Witte T, Suciu S, Verhoef G et al (2001) Intensive chemotherapy followed by allogeneic or autologous stem cell transplantation for patients with myelodysplastic syndromes (MDSs) and acute myeloid leukemia following MDS. Blood 98:2326-2331

8. Kroger N, Bornhauser M, Ehninger G et al (2003) Allogeneic stem cell transplantation after a fludarabine/busulfan-based reduced-intensity conditioning in patients with myelodysplastic syndrome or secondary acute myeloid leukemia. Ann Hematol 82:336-342

9. Ho AY, Pagliuca A, Kenyon M et al (2004) Reduced-intensity allogeneic hematopoietic stem cell transplantation for myelodysplastic syndrome and acute myeloid leukemia with multilineage dysplasia using fludarabine, busulphan, and alemtuzumab (FBC) conditioning. Blood 104:1616-1623

10. Nachtkamp K, Kundgen A, Strupp C et al (2009) Impact on survival of different treatments for myelodysplastic syndromes (MDS). Leuk Res 33:1024-1028

11. Silverman LR, Demakos EP, Peterson BL et al (2002) Randomized controlled trial of azacitidine in patients with the myelodysplastic syndrome: a study of the cancer and leukemia group B. J Clin Oncol 20:2429-2440

12. Kornblith AB, Herndon JE 2nd, Silverman LR et al (2002) Impact of azacytidine on the quality of life of patients with myelodysplastic 
syndrome treated in a randomized phase III trial: a Cancer and Leukemia Group B study. J Clin Oncol 20:2441-2452

13. Fenaux P, Mufti GJ, Hellstrom-Lindberg E et al (2009) Efficacy of azacitidine compared with that of conventional care regimens in the treatment of higher-risk myelodysplastic syndromes: a randomised, open-label, phase III study. Lancet Oncol 10:223232

14. Wijermans P SS, Baila L, Platzbecker U, Giagounidis A, Selleslag D, Labar B, Salih H, Beeldens F, Muus P, de Witte T, Lübbert M (2008) Low dose decitabine versus best supportive care in elderly patients with intermediate or high risk MDS not eligible forintensive chemotherapy: Final results of the randomized phase III study (06011) of the EORTC leukemia and German MDS study groups. Blood 112: abstr 226.

15. Steensma DP, Baer MR, Slack JL et al (2009) Multicenter study of decitabine administered daily for 5 days every 4 weeks to adults with myelodysplastic syndromes: the alternative dosing for outpatient treatment (ADOPT) trial. J Clin Oncol 27:3842-3848

16. Kantarjian H, Issa JP, Rosenfeld CS et al (2006) Decitabine improves patient outcomes in myelodysplastic syndromes: results of a phase III randomized study. Cancer 106:1794-1803

17. Fenaux P, Mufti GJ, Hellstrom-Lindberg E et al (2010) Azacitidine prolongs overall survival compared with conventional care regimens in elderly patients with low bone marrow blast count acute myeloid leukemia. J Clin Oncol 28:562-569

18. Lyons RM, Cosgriff TM, Modi SS et al (2009) Hematologic response to three alternative dosing schedules of Azacitidine in patients with Myelodysplastic Syndromes. J Clin Oncol 27:18501856

19. Silverman LR, McKenzie DR, Peterson BL et al (2006) Further analysis of trials with azacitidine in patients with myelodysplastic syndrome: studies 8421, 8921, and 9221 by the Cancer and Leukemia Group B. J Clin Oncol 24:3895-3903

20. Cheson BD, Bennett JM, Kantarjian $\mathrm{H}$ et al. Report of an international working group to standardize response criteria for myelodysplastic syndromes. In Edition 2000; 3671-3674.

21. Cheson BD, Greenberg PL, Bennett JM et al (2006) Clinical application and proposal for modification of the International Working Group (IWG) response criteria in myelodysplasia. Blood 108:419-425

22. Kaminskas E, Farrell AT, Wang YC et al (2005) FDA drug approval summary: azacitidine (5-azacytidine, Vidaza) for injectable suspension. Oncologist 10:176-182

23. Aivado M, Rong A, Germing U et al (2000) Long-term remission after intensive chemotherapy in advanced myelodysplastic syndromes is generally associated with restoration of polyclonal haemopoiesis. Br J Haematol 110:884-886

24. Aul C, Runde V, Germing U, Burk M, Heyll A, Hildebrandt B, Willers R (1997) Remission rates, survival and prognostic factors in 90 patients with advanced MDS treated with intensive chemotherapy. Haematol Blood Transfus 38:918

25. Haase D (2008) Cytogenetic features in myelodysplastic syndromes. Ann Hematol 87:515-526

26. Christiansen DH, Andersen MK, Pedersen-Bjergaard J (2003) Methylation of p15INK4B is common, is associated with deletion of genes on chromosome arm $7 \mathrm{q}$ and predicts a poor prognosis in therapy-related myelodysplasia and acute myeloid leukemia. Leukemia 17:1813-1819

27. Knipp S, Hildebrand B, Kundgen A et al (2007) Intensive chemotherapy is not recommended for patients aged $>60$ years who have myelodysplastic syndromes or acute myeloid leukemia with high-risk karyotypes. Cancer 110:345-352

28. Hackanson B, Robbel C, Wijermans P, Lubbert M (2005) In vivo effects of decitabine in myelodysplasia and acute myeloid leukemia: review of cytogenetic and molecular studies. Ann Hematol 84(Suppl 1):32-38
29. Ruter B, Wijermans P, Claus R et al (2007) Preferential cytogenetic response to continuous intravenous low-dose decitabine (DAC) administration in myelodysplastic syndrome with monosomy 7. Blood 110:1080-1082, author reply 1083

30. Raj K, John A, Ho A et al (2007) CDKN2B methylation status and isolated chromosome 7 abnormalities predict responses to treatment with 5-azacytidine. Leukemia 21:1937-1944

31. Ravandi F, Issa J-P, Garcia-Manero G et al (2008) Hypomethylating therapy in patients with AML and high-risk MDS and chromosome 5 and 7 abnormalities is associated with an improved outcome compared to conventional chemotherapy. Blood 112:2955a

32. Lim Z, Ho AYL, Samuel J et al (2007) Outcomes of MDS patients with chromosome 7 abnormalities treated with 5-Azacytidine. Blood 110:1449a

33. Mufti GJ FP, Hellström-Lindberg E, Santini V, List AF,Gore S, Seymour JF, Silverman LR, Backstrom J, Beach CL (2008) Treatment of high-risk MDS patients (pts) with -7/del(7q) with azacitidine (AZA) versus conventional care regimens (CCR): Effects on overall survival (OS). J Clin Oncol 26: abstr 7033.

34. Mufti GJ G-MG, Hovarth N, Lim Z, Quesnel B, Leone G, Bennett J, Sanz G, McKenzie D, Backstrom J, Beach CL (2008) Prolonged survival in higher-risk myelodysplastic syndrome (MDS) patients with -7/Del(7q) treated with azacitidine (AZA). Haematologica 93: abstr. 0928.

35. Robertson KD (2001) DNA methylation, methyltransferases, and cancer. Oncogene 20:3139-3155

36. Christman JK (2002) 5-Azacytidine and 5-aza-2'-deoxycytidine as inhibitors of DNA methylation: mechanistic studies and their implications for cancer therapy. Oncogene 21:5483-5495

37. Stresemann C, Lyko F (2008) Modes of action of the DNA methyltransferase inhibitors azacytidine and decitabine. Int $\mathrm{J}$ Cancer 123:8-13

38. Silverman LR, Fenaux P, Mufti GJ et al (2008) The effects of continued Azacitidine (AZA) treatment cycles on response in higher-risk patients (Pts) with myelodysplastic syndromes (MDS). Blood 112:227a

39. Müller-Thomas C, Schuster T, Peschel C, Götze KS (2009) A limited number of 5-azacitidine cycles can be effective treatment in MDS. Ann Hematol 88:213-219

40. Borthakur G, Ahdab SE, Ravandi F et al (2008) Activity of decitabine in patients with myelodysplastic syndrome previously treated with azacitidine. Leuk Lymphoma 49:690-695

41. Platzbecker U AC, Ehninger G, Giagounidis A (2009) Reduction of 5-azacitidine induced skin reactions in MDS patients with evening primrose oil. Annals of Hematology.

42. Graef T, Kuendgen A, Fenk R, Zohren F, Haas R, Kobbe G (2007) Successful treatment of relapsed AML after allogeneic stem cell transplantation with azacitidine. Leuk Res 31:257-259

43. Lubbert M, Bertz H, Wasch R et al. Efficacy of a 3-day, low-dose treatment with 5-azacytidine followed by donor lymphocyte infusions in older patients with acute myeloid leukemia or chronic myelomonocytic leukemia relapsed after allografting. Bone Marrow Transplant 2009; in press.

44. Jabbour E, Giralt S, Kantarjian H, Garcia-Manero G, Jagasia M, Kebriaei P, de Padua L, Shpall EJ, Champlin R, de Lima M (2009) Low-dose azacitidine after allogeneic stem cell transplantation for acute leukemia. Cancer 115:1899-1905

45. Platzbecker U, Radke J, Kiani A et al (2008) 5-azacitidine treatment of imminent relapse defined by decreasing donor CD34+ progenitor subset chimerism in patients with CD34+ high-risk myelodysplastic syndromes (MDS) or Acute Myeloid Leukemia (AML) after allogeneic stem cell transplantation. Blood 112:2143a

46. Kuendgen A, Schmid M, Schlenk R et al (2006) The histone deacetylase (HDAC) inhibitor valproic acid as monotherapy or in combination with all-trans retinoic acid in patients with acute myeloid leukemia. Cancer 106:112-119 
47. Soriano AO, Yang H, Faderl S et al (2007) Safety and clinical activity of the combination of 5-azacytidine, valproic acid, and alltrans retinoic acid in acute myeloid leukemia and myelodysplastic syndrome. Blood 110:2302-2308

48. Silverman LR, Verma A, Odchimar-Reissig R et al (2008) A phase I trial of the epigenetic modulators vorinostat, in combination with Azacitidine (azaC) in patients with the Myelodysplastic Syndrome (MDS) and Acute Myeloid Leukemia
(AML): a study of the New York cancer consortium. Blood $112: 3656 \mathrm{a}$

49. Voso MT, Santini V, Finelli C et al (2009) Valproic acid at therapeutic plasma levels may increase 5-Azacytidine efficacy in higher risk myelodysplastic syndromes. Clin Cancer Res 15:5002-5007

50. Sekeres MA, List AF, Cuthbertson D et al (2008) Final results from a phase I combination study of Lenalidomide and Azacitidine in patients with higher-risk myelodysplastic syndromes (MDS). Blood 112:221a 